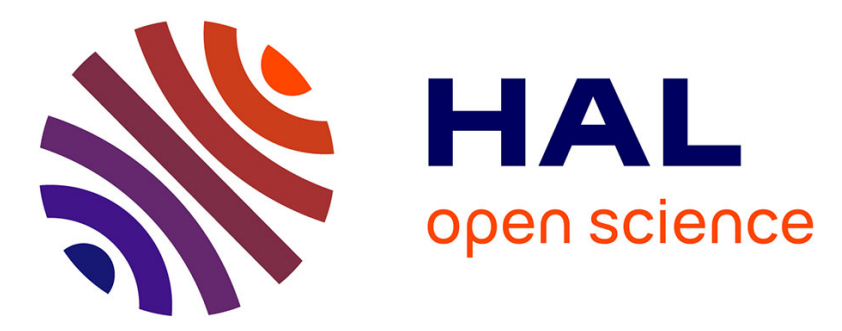

\title{
New insight into the modulated photocurrent technique using 2D full numerical simulations
}

Raphaël Lachaume, Christophe Longeaud, Jean-Paul Kleider

\section{To cite this version:}

Raphaël Lachaume, Christophe Longeaud, Jean-Paul Kleider. New insight into the modulated photocurrent technique using 2D full numerical simulations. physica status solidi (a), 2016, 213 (7), pp.1848 - 1855. 10.1002/pssa.201532969 . hal-01297095

\section{HAL Id: hal-01297095 \\ https://hal.science/hal-01297095}

Submitted on 24 Mar 2020

HAL is a multi-disciplinary open access archive for the deposit and dissemination of scientific research documents, whether they are published or not. The documents may come from teaching and research institutions in France or abroad, or from public or private research centers.
L'archive ouverte pluridisciplinaire HAL, est destinée au dépôt et à la diffusion de documents scientifiques de niveau recherche, publiés ou non, émanant des établissements d'enseignement et de recherche français ou étrangers, des laboratoires publics ou privés. 


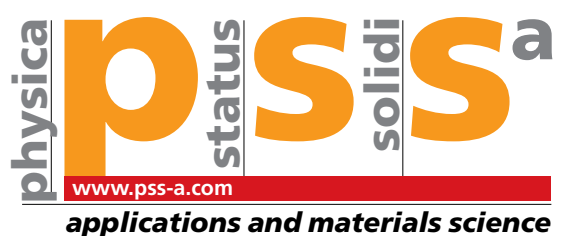

\title{
New insight into the modulated photocurrent technique using 2D full numerical simulations
}

\author{
Raphaël Lachaume*, Christophe Longeaud, and Jean-Paul Kleider \\ GeePs (Group of Electrical Engineering - Paris), UMR CNRS 8507, CentraleSupélec, Univ. Paris-Sud, Université Paris-Saclay, \\ Sorbonne Universités, UPMC Univ. Paris 06, 3 \& 11 rue Joliot-Curie, Plateau de Moulon, 91192 Gif-sur-Yvette Cedex, France
}

Received 15 November 2015, revised 25 February 2016, accepted 25 February 2016

Published online 16 March 2016

Keywords amorphous materials, density of states, modulated photocurrent, numerical simulations, silicon, thin films

*Corresponding author: e-mail raphael.lachaume@geeps.centralesupelec.fr, Phone: +33-(0)1-69851665, Fax: +33-(0)1-69418318

The modulated photocurrent technique has been used for a few decades now to provide information on the localized density of states (DOS) in semiconductor thin films. In previous studies, a simplified analytical treatment has been performed in 1D to derive equations that one has been using to reconstruct the DOS located near the majority carrier band. In these former studies, all the physical quantities were considered as constant within the sample depth, however, we know that it is not the case especially in the presence of a strong gradient of photogeneration in some materials. In this paper, for the first time, we show results of 2D simulations of the modulated photocurrent (MPC) experiment in order to study the effect of vertical photogeneration inhomogeneity on the MPC and to discuss the validity of the $1 \mathrm{D}$ assumption in more realistic cases. Thanks to these 2D simulations, new insight is gained in the understanding of the MPC experiment. Indeed, we show that we can explain and reproduce some of the experimental behaviors that the 1D theory failed to predict. Hydrogenated amorphous silicon (a-Si:H) is taken as a reference material for demonstration purposes, but this $2 \mathrm{D}$ approach can also be extended to other semiconductor materials and other types of inhomogeneities.
1 Introduction The modulated photocurrent (MPC) technique has proved to be a powerful tool to investigate the density of localized states (DOS) in the band gap of semiconductor thin films. It has been widely used to study disordered semiconductors such as hydrogenated amorphous silicon (a$\mathrm{Si}: \mathrm{H})$ [1-7] but not only. The MPC has also been applied to a large variety of materials such as (i) lead sulfide $(\mathrm{PbS})$ quantum dot arrays [8], (ii) antimony sulfide $\left(\mathrm{Sb}_{2} \mathrm{~S}_{3}\right)$ [9], (iii) copper gallium selenium compounds (CGSe) [10], (iv) phase change alloys like germanium telluride $(\mathrm{GeTe})[11]$ or germanium tin telluride (GeSnTe) [12], (v) cadmium telluride (CdTe) [13], and (vi) organic semiconductors like pentacene films $[14,15]$.

In previous works, two regimes were identified: the highfrequency regime (HF) where the MPC is determined by the trapping and release of carriers producing a significant phase shift of the MPC relative to the excitation, and the low-frequency regime (LF) where the MPC is determined by the recombination of carriers with a very low phase shift [3]. Each regime was analyzed in order to describe the phase shift and amplitude of the MPC and to reconstruct the DOS from the frequency and temperature dependence of these quantities [6]. However, these analyses relied on a simplified analytical treatment of the transport equations. In particular, it was assumed that all physical quantities under light (like the generation rate, the free carrier concentrations, etc.) were homogeneous within an effective depth related to the light absorption. This means that the true profiles of generation rate and carrier densities were not considered. We here present for the first time the full calculation of the MPC without any simplifying assumption using $2 \mathrm{D}$ numerical simulations. This calculation is compared to the previous analyses of the MPC and to experimental results. Particular emphasis is put on the transition between the HF and LF regimes and on the DOS reconstruction in both regimes.

\section{Principle of MPC and interest of this work}

2.1 Measurement procedure A typical MPC experiment consists in measuring the photo-response of a semi- 


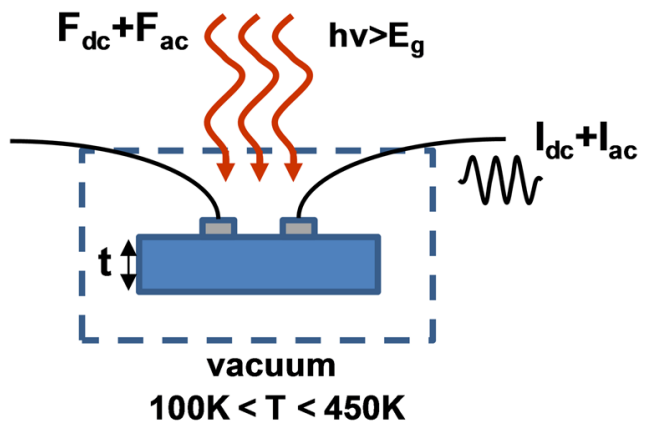

Figure 1 Simplified sketch of the MPC measurement using photon energies, $h v$, larger than the band-gap energy of the semiconductor under test, $E_{\mathrm{g}}$.

conductor sample fitted with two coplanar electrodes and submitted to a dc bias and to a modulated monochromatic light, as depicted in Fig. 1. As an illustration, we here take a $2 \mu \mathrm{m}$ thick undoped hydrogenated amorphous silicon (a$\mathrm{Si}: \mathrm{H})$ sample with $1 \mathrm{~cm}$ long top $\mathrm{Al}$ electrodes separated by $1 \mathrm{~mm}$.

After having checked the good ohmic properties of the sample, a fixed dc voltage of $100 \mathrm{~V}$ is applied between the two electrodes resulting in a constant electric field $\xi$ of $1000 \mathrm{~V} \mathrm{~cm}^{-1}$. The wavelength $\lambda$ of the light-emitting diode (LED) used to illuminate the sample should be adapted to the absorption spectrum of the film, and is fixed to $660 \mathrm{~nm}$ in this study. A small ac component - typically a sinusoidale function of time of angular frequency $\omega$ - is added to the $\mathrm{dc}$ bias of the LED resulting in a modulated incident photon flux with dc and ac components $F_{\mathrm{dc}}$ and $F_{\mathrm{ac}}$, with $F_{\mathrm{ac}} \ll F_{\mathrm{dc}}$ and $1 \times 10^{12} \mathrm{~cm}^{-2} \mathrm{~s}^{-1} \leq F_{\mathrm{dc}} \leq 1 \times 10^{16} \mathrm{~cm}^{-2} \mathrm{~s}^{-1}$. A lock-in amplifier is then used to measure the modulus of the MPC, $\left|I_{\text {ac }}\right|$, and the phase shift, $\phi$, referred to the LED signal. To obtain a wide DOS spectroscopy, the sample is put under vacuum in a cryostat in order to vary the temperature, $T$, between 100 and $450 \mathrm{~K}$. For each temperature, the reference signal frequency $f=\omega / 2 \pi$ is varied between 12 and $40 \mathrm{kHz}$.

2.2 1D formulas for DOS reconstruction For the sake of clarity, in the following we will only give formulas if electrons are the majority carriers - as it is assumed for undoped a-Si:H. In this case the MPC gives information on the upper-gap DOS only, but remember that simulations in the following do compute equations in the exact form, i.e., for both majority and minority carriers.

As already said in the introduction, previous 1D analytical analyses of the MPC showed that it is possible to extract the DOS by applying two different sets of formulas, depending on whether measurements are performed in the low-frequency regime or in the high-frequency regime [6]. These regimes are delimited in the frequency scale by the cut-off angular frequency $\omega_{\mathrm{c}}$ and in the energy scale by the quasi Fermi level for trapped electrons $E_{\mathrm{tn}}$. $\omega_{\mathrm{c}}$ is equal to the emission frequency $e_{\mathrm{n}}$ of gap states located at $E_{\mathrm{tn}}$ and is given by

$$
e_{\mathrm{n}}\left(E_{\mathrm{tn}}\right)=c_{\mathrm{n}} n_{\mathrm{dc}}=\omega_{\mathrm{c}},
$$

where $c_{\mathrm{n}}$ is the electron capture coefficient and $n_{\mathrm{dc}}$ is the steady-state free electron concentration.

2.2.1 HF regime In the high-frequency regime, i.e., for $\omega>\omega_{\mathrm{c}}$, the phase shift is due to the capture and emission of electrons in empty gap states that are located around the energy level $E_{\omega n}$, where $E_{\mathrm{tn}}<E_{\omega \mathrm{n}}<E_{\mathrm{C}}, E_{\mathrm{C}}$ being the conduction band energy. $E_{\omega n}$ can be calculated from

$$
E_{\mathrm{C}}-E_{\omega \mathrm{n}}=k_{\mathrm{B}} T \ln \left(\frac{\nu_{\mathrm{n}}}{\omega}\right)
$$

where $k_{\mathrm{B}}$ is the Boltzmann constant and $v_{\mathrm{n}}$ is the electron attempt-to-escape frequency defined by $v_{\mathrm{n}}=c_{\mathrm{n}} N_{\mathrm{C}}, N_{\mathrm{C}}$ being the effective conduction band density of states.

As detailed in original papers [2,3], the DOS at $E_{\omega n}$ can then be deduced from

$$
k_{\mathrm{B}} T \frac{c_{\mathrm{n}} N\left(E_{\omega \mathrm{n}}\right)}{\mu_{\mathrm{n}}}=\frac{2}{\pi} S q \xi G_{\mathrm{ac}} \frac{\sin (\phi)}{\left|I_{\mathrm{ac}}\right|},
$$

where $\mu_{\mathrm{n}}$ is the electron mobility, $S$ the section area of the current flow, $q$ the electron charge, and $G_{\text {ac }}$ the ac photogeneration rate related to $F_{\mathrm{ac}}$.

2.2.2 LF regime In the low-frequency regime, i.e., for $\omega<\omega_{\mathrm{c}}$, also called the recombination regime, the phase shift is very low and is determined by the recombination of carriers in partially filled gap states located at $E_{\mathrm{tn}}$, which can be evaluated thanks to

$$
E_{\mathrm{C}}-E_{\mathrm{tn}}=k_{\mathrm{B}} T \ln \left(\frac{\mu_{\mathrm{n}} N_{\mathrm{C}} S q \xi}{\left|I_{\mathrm{dc}}\right|}\right),
$$

where $I_{\mathrm{dc}}$ is the steady-state photocurrent [6].

The DOS at $E_{\mathrm{tn}}$ can then be deduced from

$$
N\left(E_{\mathrm{tn}}\right)=2 \frac{G_{\mathrm{dc}}}{k_{\mathrm{B}} T} \frac{\tan (\phi)}{\omega},
$$

where $G_{\mathrm{dc}}$ is the steady-state photogeneration rate related to $F_{\mathrm{dc}}$.

\subsection{Validity of the 1D approach?}

2.3.1 First clues in experimental data Figure 2 shows $\tan (\phi)$ values extracted from in-house experimental measurements of MPC on a $2 \mu \mathrm{m}$ thick standard coplanar sample of a-Si:H deposited on glass. The two curves are obtained by changing $F_{\mathrm{dc}}$ from $1 \times 10^{14} \mathrm{~cm}^{-2} \mathrm{~s}^{-1}$ to $1 \times 10^{15} \mathrm{~cm}^{-2} \mathrm{~s}^{-1}$ and the two arrows on the right represent the delimitation between the HF and LF regimes. The 


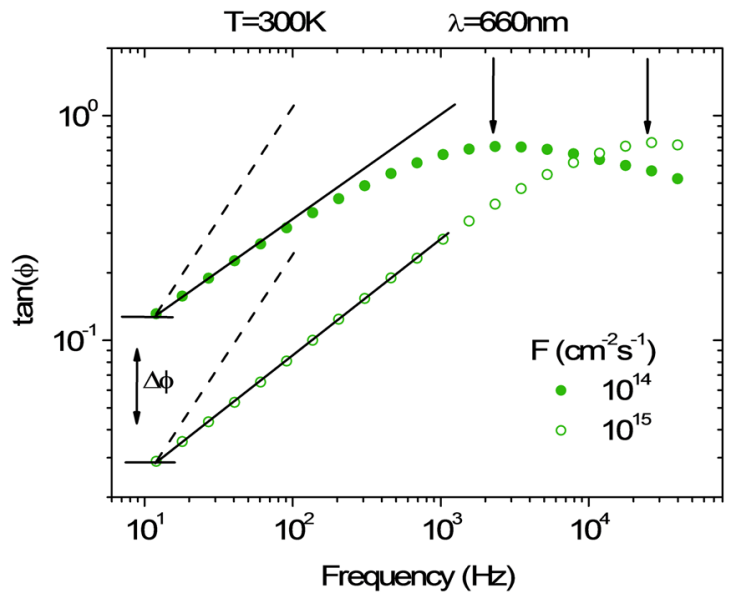

Figure 2 Phase shifts as a function of frequency for two values of dc photon flux. Data are extracted from MPC measurements performed using a red light on a $2 \mu \mathrm{m}$ thick standard coplanar sample of a-Si:H deposited on glass. The dashed lines are guides to the eye and represent lines with unity slopes. The two arrows on the right represent the delimitation between the HF and LF regimes, and are directly linked to the value of $\omega_{\mathrm{c}}$ for both values of $F . \Delta \phi$ is defined as the difference in $\tan (\phi)$ between the two curves.

latter is well recognizable in this log-log plot by the decreasing straight line formed by the data as the angular frequency decreases - a straight line which is translated downward by a given amount $\Delta \phi$ as $F$ increases. This behavior is rather in agreement with the MPC theory, however, if we take a close look at the precise values of $\Delta \phi$ and the slope of this straight line, we can see that a slight deviation exists from what Eq. (5) would have predicted. Firstly, taking $T, N\left(E_{\mathrm{tn}}\right)$, and $G_{\mathrm{dc}}$ fixed, the equation predicts a linear relationship between $\tan (\phi)$ and $\omega$ in the LF regime. In our case we can clearly see that the slope is not equal to 1 , as inferred from the deviation from the dashed line, meaning that $\tan (\phi)$ follows

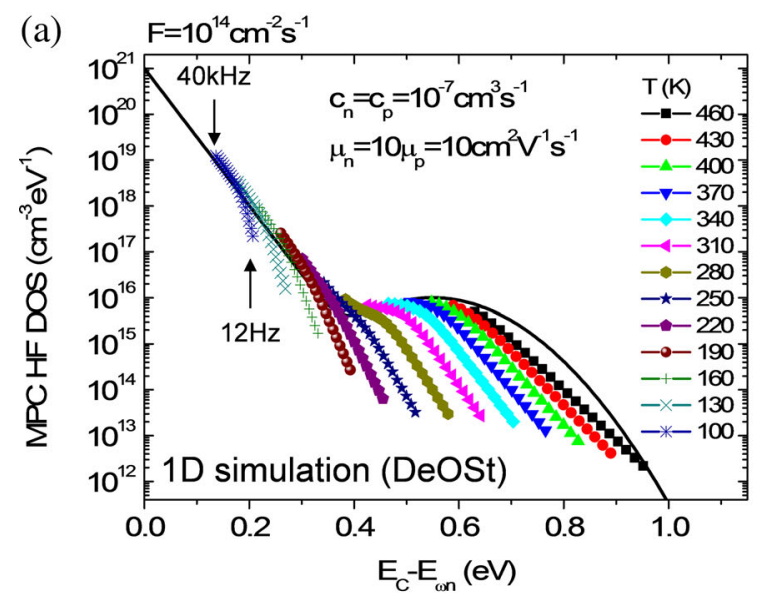

a power law instead of being proportional to $\omega$. Secondly, $\tan (\phi)$ should be strictly inversely proportional to $G_{\mathrm{dc}}$, however, in our case $\Delta \phi$ is less than a factor of 10, i.e., less than the ratio of the two photon fluxes. Both observations are clues that the 1D theory is not sufficient here to fully explain the experimental results in the LF regime. Indeed, multidimensional features such as the inhomogeneous photogeneration in the sample may not be neglected in the analysis of the MPC.

2.3.2 Current state of MPC simulation Gueunier and coworkers have already demonstrated in 1D the validity of the previous MPC formulas in HF and LF thanks to 1D simulations of the MPC experiment described in Section 2.1 using DeOSt, an in-house developed simulation program [16].

Figures $3 a$ and $b$ show such an example of standard undoped a-Si:H DOS reconstruction using DeOSt. As seen on these graphs, the introduced DOS is satisfactorily reproduced in both regimes. As explained by the MPC theory, the DOS in HF is obtained by the upper envelope of data calculated for different temperatures and frequencies. Data points deviating from this curve only indicate that $\omega$ is too low for the simulated system to be in the HF regime. For the lowest frequency data, one must use the LF formulas as it is done in Fig. $3 b$.

However, because these 1D simulations only consider the lateral transport, the vertical electron and hole distributions are constant by nature. This simplification may not be appropriate when a gradient of photogeneration exists in the sample and when the ambipolar diffusion length $L_{\mathrm{d}}$ is remarkably small compared to the film thickness. This can be the case for hydrogenated amorphous silicon thin films, for example, where $L_{\mathrm{d}}$ is of the order of $200 \mathrm{~nm}$ [17] and even below for low-grade or doped layers. To justify the use of

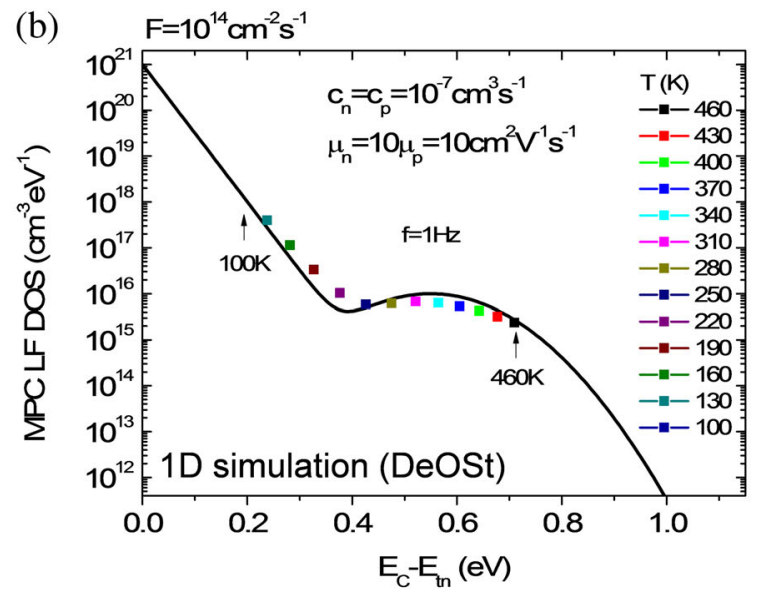

Figure 3 Example of DOS reconstruction in 1D using the MPC formulas in both HF and LF regimes from data calculated using DeOSt software to simulate the MPC of a standard undoped a-Si:H film. (a) HF DOS reconstruction. (b) LF DOS reconstruction. The black solid line is the DOS introduced in the simulations. The electron and hole capture coefficients $c_{\mathrm{n}}$ and $c_{\mathrm{p}}$ are taken equal whereas the electron mobility $\mu_{\mathrm{n}}$ is 10 times higher than the hole mobility $\mu_{\mathrm{p}}$. Note that the dark Fermi level $E_{\mathrm{F}}$ is at $0.77 \mathrm{eV}$ from $E_{\mathrm{C}}$ and that the band gap is $1.8 \mathrm{eV}$. 
(a)

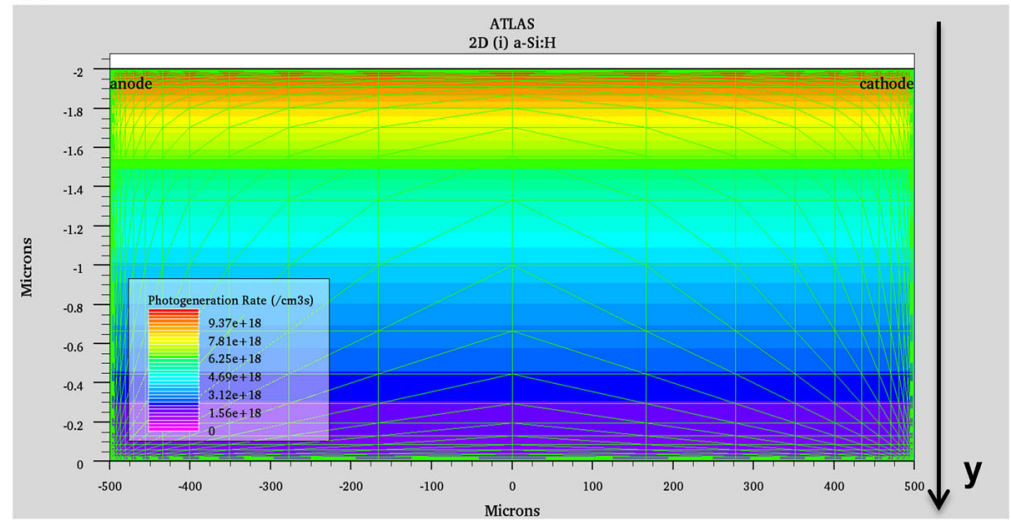

(b)

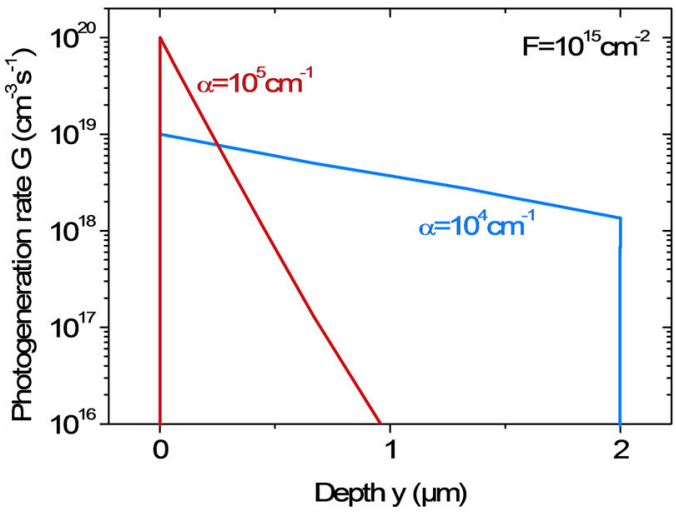

Figure 4 Modeling of the vertical photogeneration inhomogeneity in a coplanar sample submitted to the MPC technique. (a) 2D structure modeled in ATLAS. (b) Examples of simulated photogeneration profiles for different absorption coefficient values and a fixed dc photon flux.

1D formulas, it has been assumed that the MPC was mainly originating from the region close to the surface where the photogeneration rate is maximum. In DeOSt, this region is modeled as a layer of virtual thickness $t=1 / \alpha$, where $\alpha$ is the absorption coefficient, with a constant photogeneration rate $G$ given by $G=\alpha F$, where $F$ is the photon flux. Actually, to rigorously study the effects of vertical inhomogeneity on the MPC and to discuss the validity of the 1D assumption in more realistic cases, 2D simulations are needed.

3 2D modeling of the MPC experiment In this paper, we propose to simulate the MPC experiment described in Section 2.1 using the Silvaco ATLAS software [18]. The simulated 2D structure, visible in Fig. 4a, comprises a rectangular a-Si:H region of fixed length $L=1 \mathrm{~mm}$ and variable height $t$. It is contacted via ohmic boundary conditions on the left and right sides, whereas the top and bottom sides are treated as insulating boundaries. The monochromatic light source is coming from above and photogeneration inside the material is calculated at each mesh node using the Ray Tracing method [18] considering a normal incidence, a fixed $\alpha$, and no reflection. As a result, the photogeneration in the vertical direction, $G(y)$, simply follows the exponentially decaying Beer-Lambert law

$$
G(y)=G_{0} \exp (-\alpha y)
$$

$G_{0}=\alpha F$ being varied in accordance with the experimental setup possibilities. The photogeneration inhomogeneity can actually be controlled by varying the $\alpha t$ product. However, in practice, we can only vary $\alpha$ for a fixed thickness as shown in Fig. 4b. Experimentally, changing $\alpha$ means changing the wavelength of the LED because $\alpha$ is dependent on $\lambda$. In a-Si:H, for example, $\alpha$ can vary from $1 \times 10^{4} \mathrm{~cm}^{-1}$ to $1 \times 10^{5} \mathrm{~cm}^{-1}$ when the color is changed from red to blue $[19,20]$.

Regarding the electrical properties, we implemented the same models as in DeOSt. The main electrical parameters of our a-Si:H standard material are summarized in Table 1. The band diagram, the electron and hole concentrations as well as the current densities are obtained by ATLAS by numerically solving the electro-neutrality, continuity, transport (drift-diffusion), and Poisson equations self-consistently onto the mesh grid. $\phi$ and $\left|I_{\mathrm{ac}}\right|$ are post-processed using the real and the imaginary parts of the photocurrent, which are given by ATLAS by performing an ac simulation. Finally, the 1D MPC formulas given in Section 2.2 are used to reconstruct the DOS. For a full spectroscopy, all these steps need to be repeated for all $T$ and $f$ values.

\section{Results and discussion}

4.1 Constant DOS In the following, we first show simulation results considering a simplified case: i.e., we introduce a constant DOS, $N=N_{\mathrm{a}}+N_{\mathrm{d}}$, in the band-gap of a-Si:H. $N$ is the total DOS and is fixed to $1 \times 10^{15} \mathrm{~cm}^{-3} \mathrm{eV}^{-1}$; $N_{\mathrm{a}}$ and $N_{\mathrm{d}}$ are the acceptor and donor trap densities and are fixed to $3.9 \times 10^{14} \mathrm{~cm}^{-3} \mathrm{eV}^{-1}$ and $6.1 \times 10^{14} \mathrm{~cm}^{-3} \mathrm{eV}^{-1}$, respectively, positioning the dark Fermi level at a typical value for (i) a-Si:H of $\sim 0.7 \mathrm{eV}$ from the conduction band. This allows us to start the comparisons between 1D and 2D models and to validate our $2 \mathrm{D}$ model.

4.1.1 Validation of 2D calculations Actually, for a relatively small sample thickness compared to the light penetration depth $1 / \alpha$, the photogeneration can be considered as quasi-homogeneous, and there should be almost no difference between 2D and 1D simulation results.

Table 1 Main electrical parameters for standard a-Si:H.

\begin{tabular}{lll}
\hline notation & unit & value \\
\hline$E_{\mathrm{C}}-E_{\mathrm{F}}($ dark $)$ & $\mathrm{eV}$ & $0.7-0.8$ \\
$E_{\mathrm{g}}$ & $\mathrm{eV}$ & 1.8 \\
$N_{\mathrm{C}}\left(N_{\mathrm{V}}\right)$ & $\mathrm{cm}^{-3}$ & $2 \times 10^{19}\left(2 \times 10^{19}\right)$ \\
$\mu_{\mathrm{n}}\left(\mu_{\mathrm{p}}\right)$ & $\mathrm{cm}^{-2} \mathrm{~V}^{-1} \mathrm{~s}^{-1}$ & $10(1)$ \\
$c_{\mathrm{n}}\left(c_{\mathrm{p}}\right)$ & $\mathrm{cm}^{3} \mathrm{~s}^{-1}$ & $1 \times 10^{-7}\left(1 \times 10^{-7}\right)$ \\
\hline
\end{tabular}




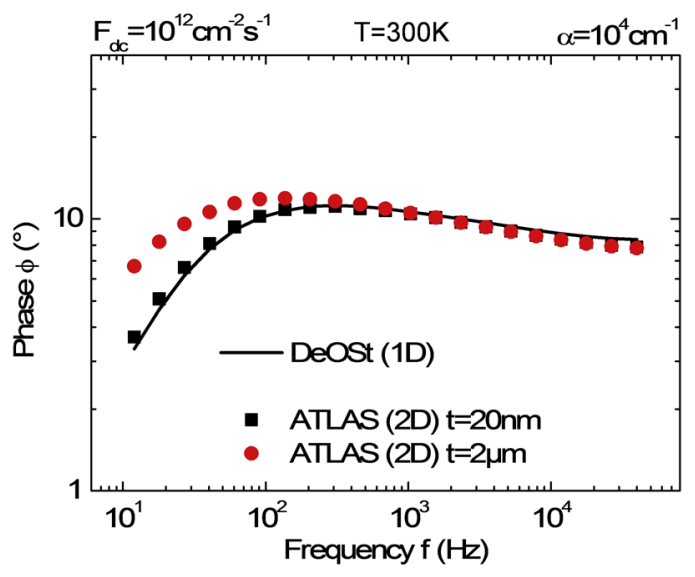

Figure 5 MPC phase shift simulated for different excitation frequencies both in 1D (DeOSt) and in 2D (ATLAS) for rather low values of photon flux $F$ and absorption coefficient $\alpha$. In the 2D model, the sample thickness, $t$, is varied from $20 \mathrm{~nm}$ to $2 \mu \mathrm{m}$.

Therefore, to validate our 2D model we first have checked in this particular case that the phase shifts calculated in 2D with ATLAS coincide well with those calculated in 1D with DeOSt. Indeed, as seen in Fig. 5, 2D simulations reproduce very well the 1D results if $t$ is fixed to $20 \mathrm{~nm}$, which is negligible compared to a present penetration depth of $1 \mu \mathrm{m}$. However, for $t=2 \mu \mathrm{m}$ photogeneration inhomogeneity is no longer negligible and some deviation does occur between both models, but at low frequencies only. In fact, above a few hundreds of hertz we no longer observe any difference between 1D and 2D calculations.

4.1.2 Effect of photon flux and absorption coefficient To check if this low-frequency discrepancy is indeed inherent to the vertical photogeneration profile, we performed additional simulations in which we increased the

(a)

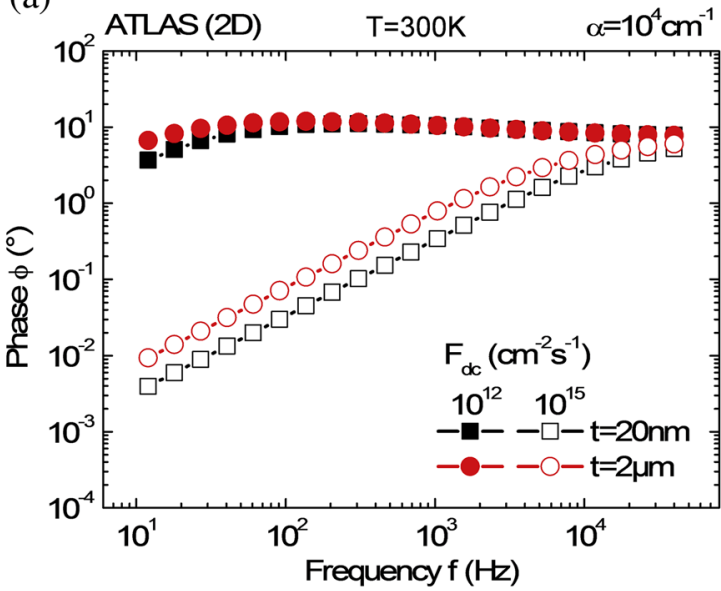

photogeneration inhomogeneity by increasing both $F$ and $\alpha$. Besides, we distinguished two different cases: (i) a quasi1D case for which $t=20 \mathrm{~nm}$ and (ii) a real 2D case where $t=2 \mu \mathrm{m}$. Both cases are compared in Fig. 6a and b: we can clearly see that the difference between quasi-1D and 2D calculations remains in the low-frequency regime and that the amplitude of this difference - for instance at a given low frequency $f=12 \mathrm{~Hz}$ - increases with $\alpha F$. To sum up, it appears that an additional contribution to the phase shift arises from the difference of photogeneration between the surface and the region deep inside the sample.

4.1.3 Impact on DOS reconstruction Before going deeper into the physical origin of this phase shift discrepancy, let us see the consequences on the DOS reconstruction using Eqs. (2)-(5). As illustrated on Fig. 7a, in HF the upper envelope of the data points calculated for a single temperature matches the constant DOS introduced in the simulations regardless of the dimensionality of the model (1D or 2D). This is explained by the fact that $\phi$ remains unaffected by the photogeneration inhomogeneity in the HF regime. This overall agreement between calculated and introduced DOS is another proof of the validity of our 2D model.

Concerning the exploitation of the $1 \mathrm{D}$ formulas in the LF regime, Fig. 7b shows that an overestimation of the DOS in $2 \mathrm{D}$ can occur. This originates from the use of Eq. (5) and from the fact that $\phi$ calculated in the LF regime is systematically higher in 2D than in 1D: the DOS overestimation in $2 \mathrm{D}$ is actually proportional to the overshoot of $\phi$ that can be seen in Fig. 6b. Whereas the error for the case $\alpha=1 \times 10^{4} \mathrm{~cm}^{-1}$ can be considered as rather small given the precision of the technique (linked to the approximations made to derive the simple form of Eq. (5)), the error in the DOS reconstruction in LF can be more than a factor of 10 for $\alpha=1 \times 10^{5} \mathrm{~cm}^{-1}$. Here, thanks to the $2 \mathrm{D}$ simulation, for the first time we demonstrate that the 1D formulas fail in the presence of significant photogeneration inhomogeneity in the sample. The physical

(b)

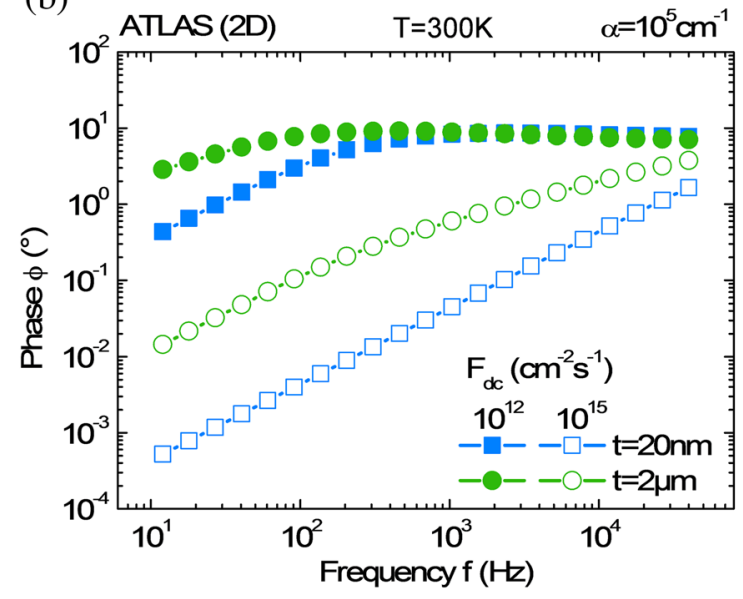

Figure 6 Effect of different vertical photogeneration profiles $(F, \alpha)$ on the MPC phase shift simulated in 2D for different excitation frequencies and for two extreme values of $t$. (a) Effect of the dc photon flux intensity for a low $\alpha$ value and (b) for a high $\alpha$ value. The lowest thickness case stands for the quasi-1D case. 
(a)

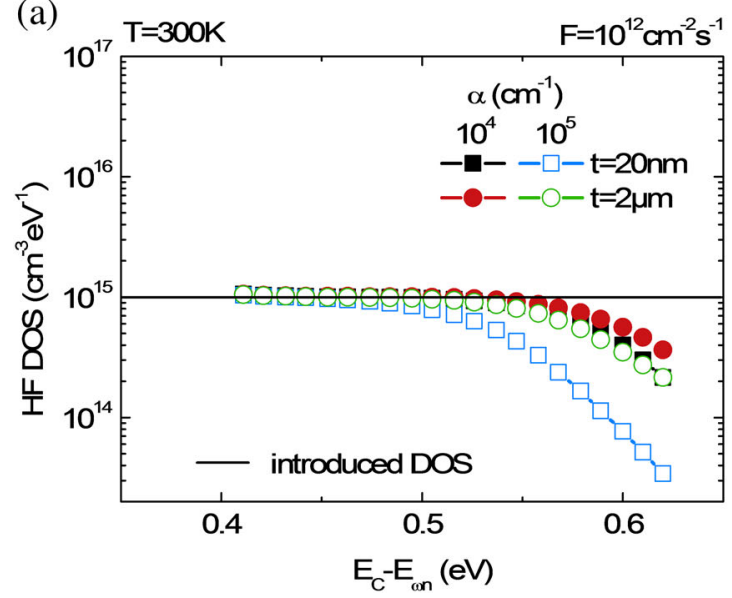

(b)

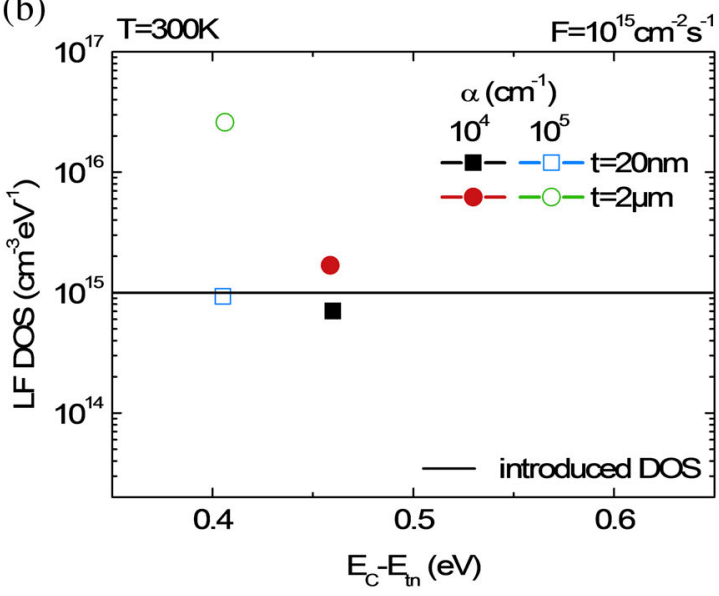

Figure 7 Reconstructed DOS using the 1D formulas for two extreme values of $t$ and $\alpha$, for a single value of $F$ and for $T=300 \mathrm{~K}$. (a) $\mathrm{HF}$ regime $-f$ is varied from $12 \mathrm{~Hz}$ (right) to $40 \mathrm{kHz}$ (left). (b) LF regime $-f$ is fixed to $1 \mathrm{~Hz}$. The lowest thickness case stands for the quasi-1D case. The black solid line is the constant DOS introduced in the simulations. The dark Fermi level is located at $0.7 \mathrm{eV}$ from the conduction band.

reason for this will be explained in detail in the following part.

4.2 Realistic DOS In the following, we will check that the previous observations are still applicable when considering a more realistic case: i.e., we now introduce a DOS that is more typical for a-Si:H (see Fig. 3), comprising two exponentially decaying band tails and Gaussian-shaped dangling bonds states. Besides, we will also compare 2D results to experimental data and provide a full physical explanation for the difference between 1D and 2D calculations.

4.2.1 Trends in simulations and in experimental data Simulations of $\phi$ at different frequencies with a more realistic DOS have also been performed with ATLAS in 2D $(t=2 \mu \mathrm{m})$ and quasi-1D $(t=20 \mathrm{~nm})$ and have been compared. Results are shown in Fig. 8a. One can draw the same conclusions as for the simpler case illustrated in 4.1, i.e., $\phi$ is systematically higher in $2 \mathrm{D}$ than in $1 \mathrm{D}$ in the LF regime whereas in the HF regime $\phi$ values always coincide. More interestingly, we can see that 2D simulations do reproduce three trends that are observed experimentally (refer to Section 2.3 and Fig. 2). Indeed, we observe that: (i) the slope in the LF regime is smaller than 1, (ii) the dependence of $\phi$ on $F$ at a fixed $\omega$ is also weaker than predicted by Eq. (5), and (iii) the transition between the HF and the LF regime is smoother. Remember that this transition is marked in $1 \mathrm{D}$ by the previously defined cut-off angular frequency $\omega_{\mathrm{c}}$. Because $\omega_{c}$ is dependent on the majority carrier concentration, (a)

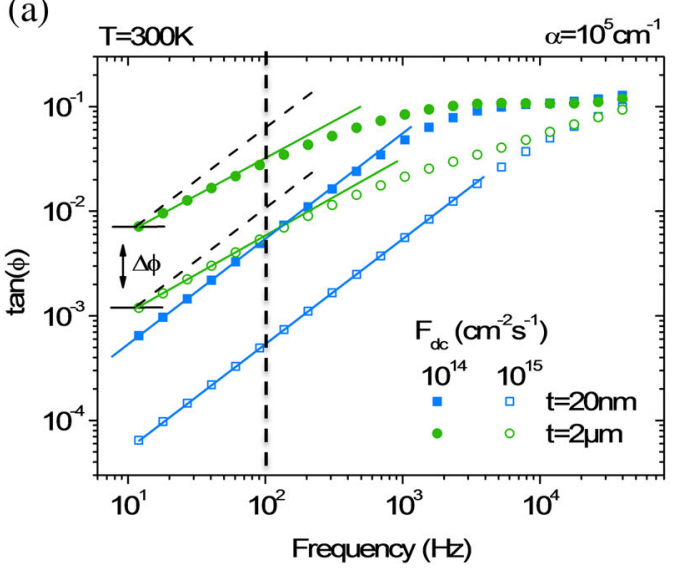

(b)

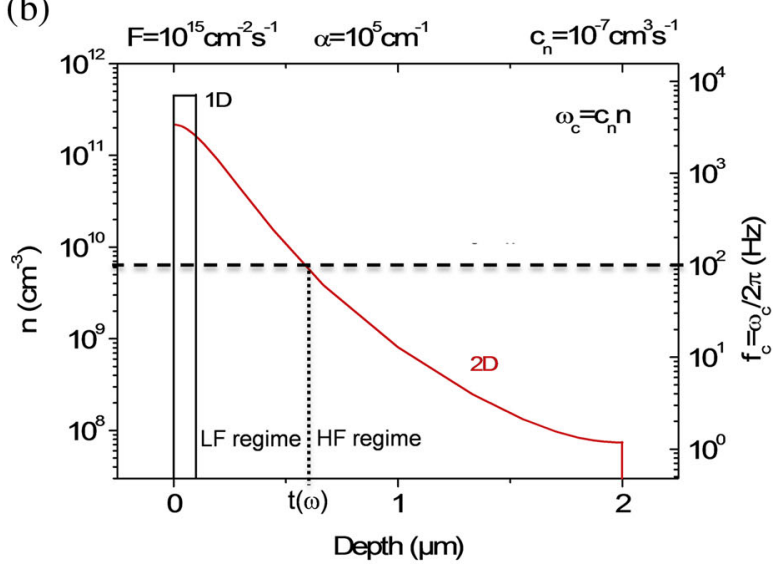

Figure 8 Comparison of the quasi-1D and 2D simulations of the MPC in case of a realistic DOS in the band gap of (i) a-Si:H and for a high absorption coefficient. (a) Phase shift as a function of excitation frequency for two different dc photon fluxes and two sample thicknesses. The lowest thickness corresponds to the quasi-1D case. (b) Electron concentration profiles as a function of depth for both quasi-1D and 2D cases. Each concentration value on the left axis corresponds to a given cut-off frequency on the right axis, obtained using Eq. (1). For a given frequency chosen equal to $100 \mathrm{~Hz}$ (dashed line in (a)), the dashed line in (b) indicates the demarcation point in the sample, $t(\alpha)$, where the working regime of the MPC changes from LF to HF. 
(a)

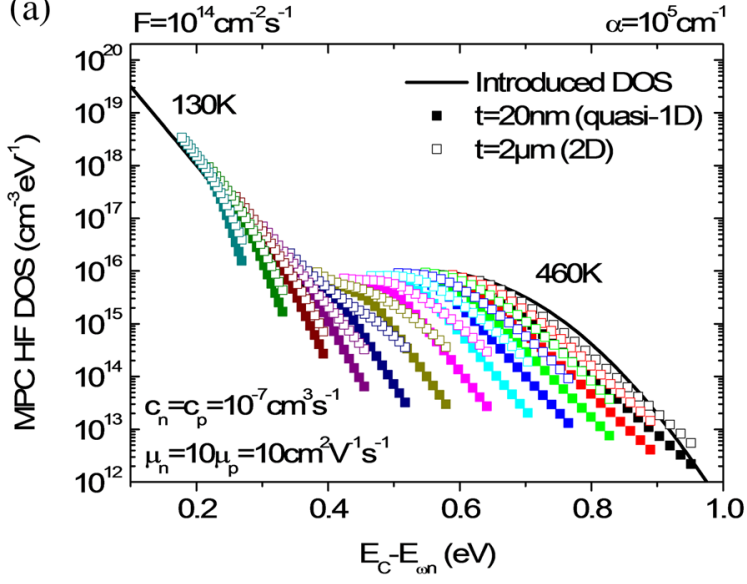

(b)

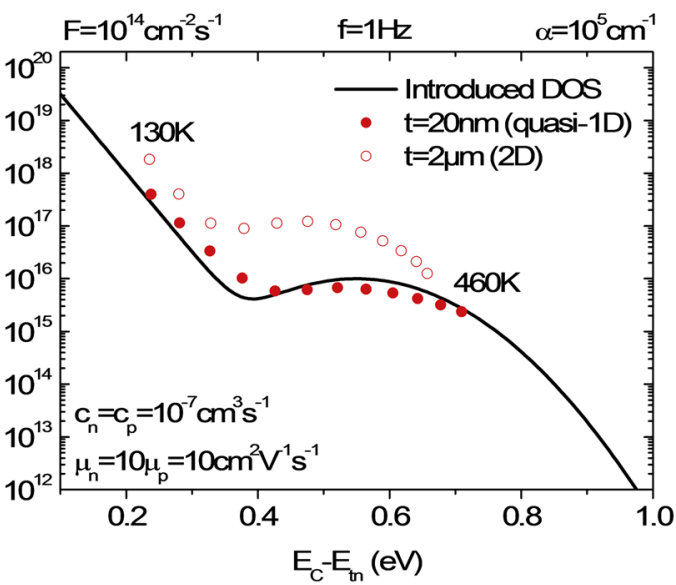

Figure 9 Reconstructed DOS using the 1D formulas for two extreme values of $t$ (quasi-1D and 2D cases), for a single value of $F$ and for $T$ ranging from 130 to $460 \mathrm{~K}$. (a) $\mathrm{HF}$ regime $-f$ is varied from $12 \mathrm{~Hz}$ (right) to $40 \mathrm{kHz}$ (left). (b) $\mathrm{LF}$ regime $-f$ is fixed to $1 \mathrm{~Hz}$. The lowest thickness case stands for the quasi-1D case. The black solid line is the constant DOS introduced in the simulations. The dark Fermi level is located at $0.77 \mathrm{eV}$ from the conduction band.

it is actually possible to explain the physical origin of this latter behavior by looking at the carrier density profile as a function of depth in the simulated sample.

As Fig. 8b shows, in quasi-1D, the electron concentration is invariant whereas in 2D, it is slightly deviating from a strict exponential decay because of the effect of diffusion. Using Eq. (1), the equivalent frequency corresponding to each carrier density value is then represented on the right axis. If we now look at the dashed line corresponding to an arbitrary excitation frequency $f=\omega / 2 \pi=100 \mathrm{~Hz}$, we see that the 1D electron concentration is above this line. This means that for this precise value of excitation frequency all states are in the same regime, i.e., the LF one. As a consequence, in the same frequency area in Fig. 8a, $\tan (\phi)$ follows a straight line with a unity slope. However, in 2D, we can see that the dashed line intercepts the electron concentration profile in the middle of the sample at a given depth denoted $t(\omega)$. Generally speaking, this means that surface states located between $y=0$ and $y=t(\omega)$ are in the LF regime, whereas deeper states located between $y=t(\omega)$ and $y=t$ are still in the HF regime thus adding a non-negligible contribution to the phase-shift. This is our explanation for the smooth transition from HF to LF but also for the phase shift overshoot systematically visible in our 2D simulations.

4.2.2 DOS reconstruction To finally conclude on the ability of the 1D formulas to provide a reliable reconstruction of the DOS, we show a last example of application of Eqs. (2)-(5) on a known pathological but realistic case where photogeneration is really inhomogeneous. Results are given in Fig. 9a and b for the HF and LF regimes, respectively.

Not surprisingly, we observe that the upper envelope of the simulated data well reproduce the introduced DOS in each case of sample thickness. Practically speaking, this means that despite a non-uniform photogeneration in the sample, it is possible to find a reasonable value of the excitation frequency for which the whole sample is behaving in the HF regime.

Concerning the calculations in the LF regime, we still observe for the realistic 2D case that the reconstructed DOS is systematically higher than the introduced one. As previously explained, this comes from the region in the sample deeper than $t(\omega)$ which is still behaving in the HF regime and which leads to a much higher value of $\phi$ than the 1D MPC theory would predict. Actually, it is worth mentioning that it is experimentally difficult to measure the low phase shifts arising at low frequencies and for this reason it is very hard to find an experimental excitation frequency for which the whole sample is behaving fully in the recombination regime.

Moreover, it turns out that the carrier diffusion effect that has not been discussed so far in this paper can also have a major impact on the DOS reconstruction. Indeed, the diffusion of surface electrons deeper in the sample leads to a decrease of the mean electron concentration in the $\mathrm{LF}$ region $(0<y<t(\omega))$, as it is visible in Fig. 8b. As a consequence, the value of $G_{\mathrm{dc}}$ used in Eq. (5) is overestimated and adds an error to the reconstruction of the DOS. Furthermore, looking again at Fig. 9b, we can see that the DOS overestimation is temperature dependent: it is less harmful when $T$ decreases. Actually, this can be explained as follows: when the temperature decreases, $E_{\mathrm{tn}}$ goes toward the conduction band, $N\left(E_{\mathrm{tn}}\right)$ increases, and in turn $L_{\mathrm{d}}$ decreases so that the effect of diffusion is less pronounced.

5 Conclusions This paper presents the first results of 2D simulations of the MPC experiment. These simulations are aimed at checking the validity of the well-known formulas obtained from the simplified 1D analysis of the MPC for reconstructing the DOS in cases where the photogeneration is not homogeneous in the sample, which is likely to occur in reality. 
It is demonstrated that the reconstruction in the HF regime works very well. However, very strong errors can occur in the transition between the HF and LF regimes and in the reconstruction of the DOS from the LF regime. In addition, our simulation results nicely reproduce the previous experimental observation that the dependence of the phase shift upon the excitation frequency in LF was weaker than predicted by the simplified analytical approach. We also show that the approximate analytical approach systematically underestimates the phase shift values in the LF regime. This discrepancy increases with $\alpha t$ and can yield errors of more than a factor of 10 in the DOS reconstructed from the LF formulas. This is interpreted by the contribution to the MPC originating from regions in the sample that are deeper than $t(\omega)>1 / \alpha$ from the illuminated surface and that are not working in the recombination regime, thus adding a nonnegligible contribution to the phase shift. We also show that the carrier diffusion effect has to be taken into account to properly reconstruct the DOS in the LF regime.

In conclusion, this work is an original extension of the MPC analysis. It brings new insight into the physics of the modulated photocurrent allowing one to avoid misinterpretations of experimental data such as overestimated reconstructed DOS values. Hydrogenated amorphous silicon (a$\mathrm{Si}: \mathrm{H}$ ) is taken as a reference material for demonstration purposes, but this $2 \mathrm{D}$ approach could and should also be applied to other semiconductor materials for which inhomogeneity effects can be important.

Acknowledgements The author would like to acknowledge Dr. Marie Gueunier-Farret for fruitful discussions on the MPC experiment and CNRS for financial support. This work was also partly supported by the French National Research Agency within APOCALYPSO project $\mathrm{N}^{\circ}$ ANR-13-PRGE-0003-02.

\section{References}

[1] H. Oheda, J. Appl. Phys. 52(11), 6693-6700 (1981).

[2] R. Brüggemann, C. Main, J. Berkin, and S. Reynolds, Philos. Mag. B 62(1), 29-45 (1990).
[3] C. Longeaud and J. P. Kleider, Phys. Rev. B 45, 11672-11684 (1992).

[4] C. Longeaud and J. P. Kleider, Phys. Rev. B 48, 8715-8741 (1993).

[5] P. Kounavis, Phys. Rev. B 64, 045204 (2001).

[6] M. E. Gueunier, C. Longeaud, and J. P. Kleider, Eur. Phys. J. Appl. Phys. 26(2), 75-85 (2004).

[7] M. Pomoni and P. Kounavis, Philos. Mag. 94(21), 2447-2471 (2014).

[8] P. T. Erslev, H. Y. Chen, J. Gao, M. C. Beard, A. J. Frank, J. van de Lagemaat, J. C. Johnson, and J. M. Luther, Phys. Rev. B 86(15), 155313 (2012).

[9] A. Darga, D. Mencaraglia, C. Longeaud, T. J. Savenije, B. ORegan, S. Bourdais, T. Muto, B. Delatouche, and G. Dennler, J. Phys. Chem. C 117(40), 20525-20530 (2013).

[10] A. Krysztopa, M. Igalson, Y. Aida, J. K. Larsen, L. Guetay, and S. Siebentritt, J. Appl. Phys. 110(10), 103711 (2011).

[11] C. Longeaud, J. Luckas, D. Krebs, R. Carius, J. Klomfass, and M. Wuttig, J. Appl. Phys. 112(11), 113714 (2012).

[12] J. Luckas, A. Piarristeguy, G. Bruns, P. Jost, S. Grothe, R. M. Schmidt, C. Longeaud, and M. Wuttig, J. Appl. Phys. 113(2), 023704 (2013)

[13] B. H. Hamadani, J. Roller, P. Kounavis, N. B. Zhitenev, and D. J. Gundlach, Sol. Energy Mater. Sol. Cells 116, 126-134 (2013).

[14] S. Gorgolis, A. Giannopoulou, and P. Kounavis, J. Appl. Phys. 113(12), 123102 (2013).

[15] N. Vagenas, A. Giannopoulou, and P. Kounavis, J. Appl. Phys. 117(3), 033105 (2015).

[16] DeOSt: A computer simulation program dedicated to the simulation of several material characterization techniques, developed at GeePs laboratory, http://lgep.geeps.centralesupelec. fr/index.php?page =scm-logiciels, 2010.

[17] A. FathAllah, F. Ventosinos, and C. Longeaud, J. Phys.: Conf. Ser. 558(1), 012011 (2014).

[18] Silvaco atlas manual, 2015.

[19] Y. Hishikawa, N. Nakamura, S. Tsuda, S. Nakano, Y. Kishi, and Y. Kuwano, Jpn. J. Appl. Phys. 30(5R), 1008 (1991).

[20] R. Street, Technology and Applications of Amorphous Silicon, Springer Ser. Mater. Sci., Vol. 37 (Springer-Verlag, Berlin, Heidelberg, 2000). 